\title{
EL ASEGURAMIENTO DE CALIDAD Y LOS PROCESOS DE CERTIFICACION EN EL AGRO COLOMBIANO: BONDADES Y DESAFIOS
}

\section{QUALITYASSURANCEINCERTIFICATIONPROCESSESANDTHE COLOMBIAN AGRICULTURAL SECTOR: KINDNESS AND CHALLENGES}

\author{
José A. Cleves $\mathrm{L}^{1 *}$, Jorge A. Fonseca $\mathrm{C}^{2}$, Alfredo de J. Jarma ${ }^{3}$ \\ Recibido para publicación: Mayo 16 de 2012 - Aceptado para publicación: Marzo 192013
}

\begin{abstract}
RESUMEN
Velar por la calidad, inocuidad, preservación del ambiente y gestión de la calidad en los procesos de producción alimentaria, es actualmente, responsabilidad de los sectores agroalimentario y agropecuario. Tales parámetros constituyen una garantía en la protección de la salud humana y representan el núcleo alrededor del cual se establecen las exigencias para la producción y comercialización de alimentos; fomentando el desarrollo de modelos alternativos de producción que involucran componentes de tipo social, económico, ambiental, administrativo y normativo. Para responder efectivamente a los requerimientos de los consumidores, se han venido desarrollando actividades que permitan la regulación de los procesos de producción, transformación y distribución, a través de la implementación del Sistema de Gestión de Calidad (SGC); el cual es requisito indispensable para acceder a procesos de certificación. El objetivo de esta revisión es presentar las generalidades del SGC y su incidencia en el sector agrícola colombiano, sus componentes, normatividad y el proceso de certificación, reseñando tanto sus ventajas como sus limitaciones y desafíos.
\end{abstract}

Palabras clave: alimentos, inocuidad, calidad, certificación.

\begin{abstract}
Ensuring quality, safety, environmental preservation and management in food production processes is responsibility of both agricultural and husbandry food industries. These factors guarantee safety of human health and represent the starting point to establish requirements for production and food marketing, encouraging development of alternative production models including social, economic and environmental components. In

'Ingeniero Agrónomo M.Sc. Profesor Asociado, Investigador Universidad Pedagógica y Tecnológica de Colombia. Facultad Seccional Duitama. Escuela de Administración de Empresas Agropecuarias. Colombia, E-mail: clevesalejandro@yahoo.com

${ }^{2}$ Ingeniero Agrónomo Esp. M.Sc. Profesor Asistente, Universidad Nacional Abierta y a Distancia. UNAD, Escuela de Ciencias Agrícolas. Tunja, Colombia. Jorge.fonseca@unad.edu.co

${ }^{3}$ Ingeniero Agrónomo PhD. Profesor Titular, Investigador Universidad de Córdoba, Montería, Colombia. jarma@fca.edu.co
\end{abstract}


order to effectively fulfill consumer's requirements, activities to allow regulation of production, processing and distribution processes, through the implementation of Quality Management System (QMS) should be implemented, as a requirement to access to certification processes. The purpose of this review is to present the general aspects of QMS and its incidence on the Colombian agricultural industry, components, standards and certification process, focusing on the advantages, limitations and challenges.

Key words: foods, safety, quality, certification.

\section{INTRODUCCIÓN}

El aumento actual de losconsumidoresalrededor del mundo, sumado a la crisis alimentaria manifestada en la trasmisión de padecimientos a través de los alimentos (eg. enfermedad de las vacas locas, micotoxinas, hormonas, etc.) (Pop et al. 2011; Spiegel et al. 2013) así como la creciente especialización y globalización de los mercados, exige a los productores agrícolas y alimentarios realizar ajustes en sus sistemas de producción, buscando dar respuesta a la demanda creciente de los mercados en términos de calidad e inocuidad de alimentos (Arenas 2000). Estos últimos parámetros son los principales limitantes para el crecimiento de las exportaciones agropecuarias colombianas (Castellanos 2004; Fonseca 2007).

Por exigencia de los consumidores, en los últimos años se han incrementado los criterios de producción sostenible, que involucran criterios ecológicos o de buenas prácticas agrícolas (BPA), queincluyen la implementación del Sistema de gestión de calidad (SGC) (Contreras 2008). Dicho sistema, permite elevar el nivel competitivo de las unidades productivas, facilitando el acceso a nuevos mercados con base en el aseguramiento de la inocuidad, calidad y adecuado suministro de los alimentos; comprendiendo conceptos de manejo ambiental (Almirall et al. 2008), generando bienestar a los trabajadores rurales, a sus familias y finalmente a los consumidores (Venegas 2010). En este proceso, se potencializan las capacidades competitivas de los agricultores, propendiendo por la inclusión de una visión empresarial en el desarrollo de sus actividades (CCI 2004).

En general, las BPA comprenden tres lineamientos básicos: (1) Inocuidad; (2) sostenibilidad ambiental y salud; y (3) seguridad y bienestar laboral. El desarrollo y la aplicación de tales lineamientos permiten el crecimiento constante tanto en la productividad como en la calidad, reduciendo progresivamente los costos unitarios de producción, manteniendo la rentabilidad suficiente para retribuir los recursos invertidos y financiar adecuadamente los proyectos de modernización, mejorando la calidad ambiental de los procesos productivos, contribuyendo al bienestar y a la convivencia de la comunidad; siendo parte del análisis de los diferentes riesgos e implementando medidas oportunas de control (Ministerio de Agricultura et al. 2005).

Considerando la importancia de las BPA en los sectores participes de las cadenas productivas agronómicas y agropecuarias, así como el desarrollo actual de un sistema que propende por el mejoramiento de la inocuidad y la calidad de los alimentos; la presente revisión 
considera el estado actual del conocimiento en estas temáticas, la normatividad pertinente, así como las ventajas y desventajas en la implementación del sistema de gestión de calidad (SGC), haciendo énfasis en el caso Colombiano, del cual a la fecha, se cuenta con muy poca información referenciada.

\section{El sistema de gestión de la calidad (SGC)}

Se define como un conjunto de partes o elementos relacionados y organizados que interactúan entre sí y con el ambiente, es decir actúan como un todo para lograr un objetivo o propósito y dar respuesta a una necesidad concreta (Báez 2006; Bertalanfly 1976; Capra 1998). En tal sentido y bajo el contexto de la presente revisión, la finca o unidad productiva debe ser considerada como un Sistema Empresa Agropecuario o Agroempresa (Contreras y Restrepo 2007b).

La Agroempresa se define como una unidad con capacidad para tomar decisiones (Murcia 2007). Este sistema, es de tipo abierto que posee unas entradas en términos de energía, recursos y/o insumos que son básicos para el proceso productivo y unas salidas, que son la resultante de la interacción de los insumos o entradas, reportados en términos de productos biológicos, ambientales y/o financieros; que en últimas son la razón de ser del proceso productivo (Espejo 2009).

Un desbalance de los elementos que constituyen el sistema es una de las principales causas para reducir la calidad, rentabilidad y sostenibilidad de la actividad agrícola (Correa 2009).

\section{Gestión}

Se define como "la administración de los recursos, la movilización de voluntades y la toma de decisiones, para la consecución de los objetivos con recursos limitados en un tiempo determinado" (Contreras 2008).

En la agroempresa, la gestión se puede entender como un conjunto de actividades que involucran estrategias y medios para alcanzar los objetivos en términos de productividad, rentabilidad y sostenibilidad (Linares y Salamanca 2003). Es sin duda la capacidad que desarrolla la agroempresa para garantizar que su proceso productivo cumpla con las expectativas de los clientes, inversionistas y trabajadores (Mateos 2002).

\section{Calidad}

Es el conjunto de propiedades y características de un producto o servicio que le confieren la aptitud para satisfacer necesidades expresas o implícitas (ISO 2000a). Los aspectos de calidad hacen referencia a características físicas u organolépticas del producto (Codex alimentarius 1993). También se refiere a la calidad en el servicio, que es el resultado generado por las actividades de la interface entre el proveedor y el cliente y por las actividades internas del proveedor.

La gestión de la calidad referida a actividades internas en las agroempresas, son el conjunto de actividades que desde la dirección, determinan las políticas de calidad, como un marco de acción regulatoria sobre el cual se van a desarrollar las actividades de producción, adecuación, comercialización, transformación, transporte y distribución. (Contreras y Puentes 2009). En la gestión de la calidad, se usa como 
herramienta central el ciclo de gestión de Deming o de control de calidad PHVA: Planear, Hacer, Verificar y Actuar (Borrego 2010; ISO 2000b). Su función es desarrollar una serie de actividades al interior de la agroempresa para prevenir las causas que originan problemas en los procesos de producción, reduciendo los errores y desperdicios, manteniendo la calidad y dejando siempre como soporte evidencias escritas (Deming et al. 1989).

El SGC, se sustenta en normas que especifican un procedimiento para lograr una efectiva administración de la calidad, dando cumplimiento a una normativa, reglamento o código de conducta, que busca aumentar las capacidades o niveles competitivos en las unidades de producción. Las partes que componen el sistema de gestión son: (1) Estructura organizativa, la cual dirige y coordina el departamento de calidad y es responsable de la dirección de la empresa; (2) Planificación de la calidad; (3) Procesos de la organización y (4) Logística o recursos requeridos para la Agroempresa que aplica la calidad. EI SGC por tanto, es una estrategia competitiva que busca insertar a los sectores productivos en una posición provechosa y favorable que les permita competir con éxito en mercados altamente exigentes (Oyarzún \& Tartanac 2002).

La implementación de los sistemas de gestión de calidad, se basan principalmente en las normas ISO 9000, consensos internacionales de manejo de buenas prácticas que aseguran que una organización pueda entregar productos o servicios que cumplan con los requisitos de calidad que exige el cliente (Aggelogiannopoulos et al., 2007). Para el caso agropecuario colombiano caben destacar la Norma ISO 9001: 2000 (Gallardo 2000); La Norma ISO 9004; La Norma ISO 19011; La Norma ISO 14000; La Norma ISO 18000 también conocida como OHSAS y La Norma ISO 22000 (Codex alimentarius et al. 2006; Belibagli et al. 2009).

\section{Componentes documentales del SGC}

La implementación del SGC se inicia con la capacitación de la agroempresa desde la gerencia hasta el último empleado; esto permite educar al personal, hacerse menos resistente a los cambios que se generen al adherir a la norma, ensamblar los procesos de manera más eficiente, así como sensibilizar a la organización para crear un sistema gerencial moderno (UTZ Certified 2009), que sea capaz de adaptarse rápidamente al requerimiento del cliente. Posteriormente se realiza el proceso de documentación, mientras que la Gerencia, trabaja con elementos de la planificación estratégica, y deberá estar permanentemente monitoreando el proceso de implementación del SGC. Dentro de los componentes documentales que ayudan al proceso de implementación se incluyen: (Fonseca y Cleves 2009; Correa 2009).

\section{Manual de Calidad (MC)}

Es un documento donde se enuncia el compromiso con la calidad de los productos, en general describe el sistema de calidad de la empresa (Contreras 2008). El manual establece los lineamientos a seguir en las actividades de producción para lograr los estándares de calidad requeridos por la organización y sus clientes. Los cuales suelen estar establecidos en normas o códigos de cumplimiento, que la organización implementa con fines de certificación. (UTZ 
Certified 2009). De la misma forma el manual de calidad establece el funcionamiento de la organización, la política de calidad, su estructura administrativa, los objetivos y alcances, responsabilidades, funciones de sus integrantes y procesos administrativos entre otros aspectos. Es por tanto, la columna vertebral del SGC y permite a los trabajadores de la Agroempresa entender y dimensionar las reales intensiones en términos de calidad y competitividad (Fonseca y Cleves 2010).

\section{Manual de Procedimientos (MP)}

Este documento orienta sobre la forma en que se instrumentará o aplicará la política de calidad en las diferentes áreas que componen la agroempresa y constituye la guía de cómo se deben llevar a cabo las diferentes actividades por parte del personal tanto técnico, administrativo, como de producción. Los procedimientos deben estar escritos y al alcance de quienes necesitan aplicarlos, se presentan de manera detallada, especificando metodologías y herramientas a utilizar (Contreras y Restrepo 2007b).

\section{Manual de Documentación (MD)}

Contiene el listado de los documentos que se usan dentro del SGC con sus respectivos códigos, los cuales obedecen al proceso de sistematización interno, además de poseer los formatos que se requieren para consignar la información de las actividades realizadas, con los cuales se construye y configura la trazabilidad del producto. Para estructurar la documentación de todo sistema de gestión es necesario tener en cuenta los requisitos que contempla la norma ISO 9001 (Cleves et al. 2011; Montañez 2009).
ProcesodeimplementacióndelSGCconfines de certificación

La certificación es un proceso de inspección y verificación realizado por entidades independientes y especializadas denominadas Empresas Certificadoras (Caplam 2006). Ellas se encargan de evaluar el cumplimiento de requisitos específicos, los cuales se comparan frente a referentes definidos como son las normas técnicas, reglamentos, protocolos o códigos de conducta. El proceso se inicia cuando las agroempresas en forma individual o como grupo de productores asociados deciden desarrollar e implementar el SGC, dando cumplimiento a una norma o protocolo que es seleccionada de acuerdo a las conveniencias comerciales o al interés en un mercado específico (Abarca y Sepúlveda 2001; GLOBALGAP 2007).

La Certificación Agropecuaria es un mecanismo que sirve para identificar el producto, asociando sus características con parámetros específicos de calidad (IFAT 2002), constituyéndose como una herramienta ineludible que facilita a las empresas la introducción de sus productos y servicios en otros mercados. Es una confirmación de manera formal y escrita (licencia o certificado) otorgada por una entidad especializada que otorga al productor o grupo de ellos, la constancia de que cumplen con las normas o especificaciones voluntarias con las cuales se habían comprometido o que están obligados a cumplir (IFAT 2002) y por tanto da la confianza de que el objeto de certificación satisface los requisitos establecidos (Fernández 2003). El proceso de certificación posee un reglamento de contratación y uso; estando acompañado de un proceso que comprende la concesión 
de la marca; la auditoria del producto, que pretende verificar los procesos administrativos, técnicos y de producción de las unidades en forma individual o grupal (Hernández 2009); y la sanción de infracciones (IFAT 2002).

\section{Normalización con fines de certificación en Colombia}

La normalización es la actividad que consiste en establecer, con respecto a problemas reales o potenciales, disposiciones para uso común y repetido, encaminadas al logro del grado óptimo de orden en un contexto dado (Peinado 2006). En general, es un instrumento de ordenamiento tecnológico, que ofrece a las sociedades y gobiernos, importantes beneficios al facilitar la adaptación de los productos, procesos y servicios a los fines a los que se destinan, protegiendo la salud y el medio ambiente, previniendo los obstáculos al comercio y facilitando la cooperación tecnológica (Contreras y Restrepo 2007a).

En Colombia los procesos de normalización con fines de certificación son vigilados por la superintendencia de Industria y Comercio a la cual se encuentran vinculados organismos de certificación como: Bcs, Controlunión, Biolatina, Biotropico entre otras, encargados de tales tareas. En particular, para el sector hortofrutícola Colombiano, las principales normas, protocolos y/o códigos de conducta que se están implementando con fines de certificación para procesos de exportación, se pueden dividir en cuatro grandes grupos (Conpes 2001; Fernández 2003):

\section{Normasbasadasen BuenasPrácticasAgrícolas (BPA)}

Las BPA son el conjunto de criterios que involucran tanto aspectos relativos a la actividad agrícola como los principios de buenas prácticas de manufactura (BPM) y buenas prácticas de higiene (BPH), las cuales son transversales en la producción. Esta unión de criterios son un sistema de aseguramiento de la inocuidad, que con enfoque preventivo se aplican a toda la cadena, reuniendo las condiciones operativas recomendadas para el mejoramiento de los métodos convencionales de producción (Contreras y Restrepo 2007a).

Dentro de este tipo de normas, se destaca la Norma GlobalGap, un protocolo para las buenas prácticas agrícolas para frutas y vegetales que se constituye en requisito de obligatorio cumplimiento para las exportaciones agropecuarias hacia la Unión Europea y Estados Unidos (Ablan 2000; Torrado 2005). Además del sistema ISO 9000, el sistema HACCP (Hazard Analysis and Critical Control Points) ha venido implementándose en los sistemas de gestión de calidad y de control de procesos (Manis 1995) tal sistema pretende identificar las amenas, los puntos críticos de control y las medidas preventivas para la implementación de un sistema de monitoreo en la producción alimentaria (Sandrou y Arvanitoyannis 2000) eliminando o reduciendo con ello los riesgos que puedan afectar la seguridad alimentaria (Codex alimentarius Commission 2003). El sistema HACCP por tanto previene los problemas de los productos, en lugar de solucionarlos una vez terminados (Rooney y Kilkelly 2002). Actualmente es común la aplicación del sistema HACCP en conjunto con la implementación conjunta de los sistemas de gestión de calidad ISO 9000, generando un sistema de calidad completo en la industria alimentaria (Pop y Pop 2006). En la actualidad los principios 
del sistema HACCP han sido ampliamente implementados en las principales potencias a nivel agroindustrial incluyendo Canadá, países pertenecientes a la Unión Europea, Australia y Nueva Zelanda, constituyéndose como uno de los sistemas de mayor aceptación y éxito (Ropkins y Beck 2000).

La norma nacional NTC 5400, proporciona los requisitos generales y las recomendaciones para el territorio Colombiano. Actualmente se trabaja en un proceso de homologación con Globalgap, para obtener el estándar Colombiagap. En nuestro país bajo esta norma están certificados 559 agricultores con un área total de 140.000 ha., mientras que en otros 100 países existen 100.000 productores certificados (Corpoica 2010). Otras normas basadas en BPA, incluyen la Norma Flor Verde y UTZ Certifield (Cáceres 2004).

\section{Normas de producción orgánica}

La Unión Europea posee la norma orgánica CEE, que permite al consumidor reconocer como orgánico productos que presentan como principal característica estar exento de residuos químicos contaminantes. Para el caso de Japón se usa la norma Japanese Agricultural Standars (JAS), que limita el uso de agroquímicos y procura mejorar la fertilidad del suelo. Y la norma orgánica de los Estados Unidos USDA NOP, (Pérez 2008), bajo la cual se pueden certificar productos agrícolas y pecuarios (Fedecacao 2010).

\section{Normas ambientales}

Se destaca la norma Rainforest Alliance (RAS.) o Bird Friendly; que evalúa criterios ambientales y sociales causados por las actividades agrícolas, que tienen que ser evaluados en los procesos de inspección, cuenta con 94 criterios, permitiendo la certificación grupal o individual de las fincas (Oyarzún y Tartanac 2002). La norma, estimula a los agricultores y productores forestales a adoptar métodos sostenibles y premia sus esfuerzos. Lidera tambiénunmovimientointernacional deturismo sostenible, atrayendo turistas responsables y protegiendo los recursos naturales locales. Por su parte, la norma Veriflora, se destaca en la agricultura sustentable y en programas de etiqueta ecológica, mediante la producción sustentable de cultivos a través del desarrollo de prácticas orgánicas para el control de plagas y enfermedades; la conservación de recursos y eficiencia energética, así como la protección de ecosistemas y manejo integral de desechos, estableciendo metas únicas de reducción de gases de efecto invernadero (Fedecacao 2010).

\section{Normas de Responsabilidad Social}

Para el sector agropecuario dentro de este grupo de normas se destacan la certificación Fair Trade o de Comercio Justo, la cual busca que el comercio este basado en el dialogo, transparencia y respeto. La norma certifica únicamente a los productores que se encuentren asociados en cooperativas, asociaciones u otro tipo de organización (FLO 2002).

La norma comercio seguro Business o Aliance for Secure Comerce (BASC), por su parte, es una alianza internacional que promueve un comercio seguro en cooperación con gobiernos y organismos internacionales. Está constituida como una organización sin ánimo de lucro, en la cual participan empresarios de todo el mundo con el propósito común de fortalecer el comercio internacional de una manera ágil y segura. Su misión principal consiste 
en eliminar la utilización de mercados lícitos para el transporte de narcóticos y contrabando (Oyarzún y Tartanac 2002).

\section{Ventajas de la implementación del SGC} La implementación del SGC introduce un cambio de paradigma en el manejo tanto de aspectos productivos, administrativo y de mercados, ya que este sistema se convierte se convierte en el pilar de los procesos de la racionalización y uso eficiente de los recurso (Ministerio de Agricultura y Corpoica 2007). El Sistema promueve la sostenibilidad de los procesos productivos, desde un enfoque económico, social y ambiental, en donde el desarrollo y el bienestar social están limitados por los niveles tecnológicos, los recursos del medio ambiente y su resiliencia frente a los efectos de la actividad humana (Portilla 2007).

Desde el punto de vista económico, el SGC motiva la generación de cambios productivos mediante un proceso de reconversión (Arenas 2000), de tal manera que el producto obtenido está en capacidad de satisfacer las exigencias de los consumidores, generando un ingreso extra, mejorando las condiciones de vida $y$ salud de los agricultores y consumidores, y a su vez, garantizando la presencia continua en los mercados.

Desde un enfoque ambiental se protege $y$ fomenta la biodiversidad, se integran corredores biológicos que estimulan distintas relaciones ecológicas, estimulando prácticas de alelopatía y de control biológico, limitando el uso de insumos de síntesis química. Los riesgos fitosanitarios se evalúan con visión integradora (Correa 2009). Se implementan programas de uso eficiente y ahorro de agua, preservando el medio ambiente (Báez 2006). El uso de las fuentes de energía es más eficiente, se fomentan prácticas de conservación y preparación de suelos. Además, optimiza y racionaliza el uso y disponibilidad de los recursos naturales desatascándose la recuperación y preservación de germoplasma nativo.

Desde el punto de vista social, se valoran los saberes ancestrales o aprendidos, se estimula la capacidad de autogestión, se promociona el liderazgo y se fomentan procesos de capacitación e integración, se impulsa la cultura del éxito (Fernández 2003). Se integra a los agricultores con los avances tecnológicos, se promueve un cambio cultural dejando atrás prácticas engañosas; se impulsa la seguridad alimentaria; se desarrollan relaciones de arraigo con las unidades productivas; y se estimula la organización comunitaria.

\section{LimitantesparalaimplementacióndelSGCen el agro Colombiano}

Después de efectuar una amplia revisión sobre el estado del arte, los autores concluimos que en nuestro país existen diversos aspectos que limitan la implementación de los sistemas de aseguramiento de la calidad, entre los más importantes se puede mencionar los siguientes:

\section{Limitación Económica}

Para la puesta en marcha del SGC y su posterior certificación, es necesario implementar en la agroempresa un proceso continuo de capacitación, conformación de equipos de evaluación y seguimiento, acompañamiento de asesores externos, ajuste y/o adecuación de la infraestructura productiva y tecnológica entre otros aspectos. Lo anterior implica la erogación de recursos económicos que debe 
ser provistos por las unidades productivas. Aunado a lo anterior en el país son escasos los estímulos o subsidios económicos para el desarrollo de estas iniciativas, prevaleciendo esfuerzos e iniciativas privadas.

\section{Limitación en el tamaño predial}

De acuerdo al interés específico por situar los productos, algunas normas exigen la delimitación de una zona de amortiguamiento, la tenencia de la tierra en Colombia se caracteriza por el micro minifundio, de tal manera que para los pequeños agricultores sea difícil dejar un área sin ningún uso específico, con un fin comercial que facilite su supervivencia.

\section{Período de reconversión}

Se refiere a un periodo de transición en la cual se reemplazan o substituyen las prácticas de producción tradicionales por aquellas actividades aceptadas por los modelos de producción orgánica. En dicho periodo la cosecha no será tenida en cuenta como orgánica, ya que como un margen de seguridad se presupone la existencia de trazas químicas en el suelo que puedan contaminar el producto final. Este periodo dependiendo del estándar o norma implementada y las condiciones iniciales del lote de terreno, varía de uno a tres años. Es requisito llevar diversos registros y evidencias del proceso productivo y de manejo.

En este período se ha reportado leves descensos en los niveles de producción, los cuales gradualmente son recuperados y posteriormente superados cuando está en pleno funcionamiento el modelo de producción orgánico, además se logran mayores ingresos por los mayores precios en el mercado de estos productos los cuales en promedio son del $30 \%$.

\section{Limitación en el mercadeo}

Por las limitaciones estructurales del mercado agropecuario en el país y los escasos canales de comercialización para este tipo de productos, no siempre se logran los sobreprecios esperados, aun es limitada la demanda de estos productos y se circunscribe a pequeños nichos de mercado. Se conocen casos algunos episodios donde productos certificados orgánicos no han tenido sobreprecio en los mercados, tal como ocurrió a un grupo de productores de papa en Ventaquemada (Boyacá) quienes por falta de demanda ofertaron su producto en plazas de mercado sin un precio diferencial con respecto a la papa convencional.

Para superar estas distorsiones del mercado es necesario que se fortalezca la cadena de productos orgánicos que lidera el Ministerio de Agricultura, estableciendo contratos forward (venta de futuros) para estos productos, introduciendo incentivos tributarios tanto a productores como a comercializadores, desarrollo de ruedas de negocio y alianzas estratégicas entre organizaciones de productos y agroindustriales así como una mayor difusión publicitaria para incentivar su consumo.

\section{Limitaciones investigativas}

En el proceso de revisión de literatura para el desarrollo del presente trabajo se pudo constatar que en Colombia y en general en Suramérica, es escaso el abordaje investigativo sobre los procesos de gestión de la calidad y los procesos de certificación agropecuaria, no existen claros resultados que demuestren que la implementación del SGC en la producción agropecuaria redunde significativamente 
en mayores niveles de competitividad (especialmente de mayores ingresos para los productores), ni tampoco en mayores niveles de sostenibilidad ambiental. Por lo que se hace urgente evaluar la incidencia de los procesos de certificación en Agroempresas con diferentes niveles tecnológicos y financiero, estableciendo el comportamiento de indicadores económicos, sociales y ambientales.

\section{Intereses de las empresas certificadoras}

Si bien es cierto que la implementación del sistema de gestión de calidad en el sector agropecuario busca optimizar el uso de recursos y estandarizar los procesos de producción con el fin de aumentar los niveles de inocuidad de la producción de bienes alimentarios. Se está haciendo a partir de modelos administrativos y tecnológicos que se han desarrollado en otras latitudes y no siempre responden a la realidad del productor agropecuario colombiano, y aún más a pequeños productores rurales (que son más del $80 \%$ de los productores agropecuarios del país), causando una serie de traumatismos y mayores costos en el proceso de implementación. De otra parte, estos procesos son excluyentes por los altos requisitos en términos tecnológicos y de infraestructura que se requieren para el proceso de certificación, dejando con escasas posibilidades a pequeños productores que no cuente con estos elementos y con los altos recursos que se requieren para su implementación. Dejando únicamente con posibilidades a aquellos con mayor capacidad de negociación, musculo financiero y habilidad para colonizar mercados.

Las empresas de certificación viene promoviendo la certificación como la etapa complementaria a la implementación del SGC, cual no necesariamente es así, pues la primera simplemente es el aval que estas dan del cumplimiento de un estándar de calidad, que les permite a estas transnacionales cuantiosos ingresos en todo el mundo, sin que ello en realidad fomenta la inocuidad de los alimentos o la preservación del medio ambiente. Es decir el que nuevamente aspectos sensibles para la población humana como alimentos sanos y medio ambiente se convierten en un jugoso negocio por disposición de los países importadores de alimentos, quienes establecen los criterios que los productores deben cumplir.

\section{CONCLUSIONES}

EI SGC es una decisión administrativa eficiente para superar dificultades productivas, que desde un punto de vista agroecológico, optimiza recursos al efectuarse una adecuada planeación en los procesos productivos. Es un proceso de mejoramiento continuo $y$ verificable del sistema de producción, que permite dar cumplimiento a las normas, códigos y/o protocolos que están exigiendo los mercados especializados a nivel nacional y mundial para asegurar la inocuidad y calidad de los alimentos, a través de la implementación de mecanismos de monitoreo de peligros, valoración y mitigación de riesgos.

El SGC es una herramienta fundamental en los procesos de planeación administrativa y técnica, que promueve la sostenibilidad de los procesos productivos, desde un enfoque administrativo, económico, social y ambiental. Sin embargo las numerosas referencias bibliográficas consultadas, no reportan resultados en cuanto a la parametrización del proceso de certificación y tan solo 
reseñan el proceso de aseguramiento de la calidad como una actividad que encarece el proceso productivo. Se requiere de la evaluación de los impactos concretos, medibles, cuantificables y verificables, que sobre las unidades productivas y sus familias eventualmente tengan la implementación de dichas normativas. Así mismo, es necesario medir el impacto del los SGC en términos de indicadores de sostenibilidad con principios de buenas prácticas agrícolas y sus eventuales efectos en los aspectos económico, ambiental y social.

La implementación del SGC es un elemento significativo para mejorar los niveles de calidad de vida de los productores y la salud de los consumidores, preservando las condiciones ambientales. El Estado Colombiano debe procurar en sus políticas agrícolas la inclusión de estímulos o subsidios a los agricultores interesados en articularse a mercados específicos, caracterizados por la alta competitividad y cumplimiento en la oferta.

\section{REFERENCIAS}

Abarca, R. y Sepúlveda, S. 2001. Ecoetiquetado: Un Instrumento para Diferenciar Productos e Incentivar la Competitividad. Cuaderno Técnico $\mathrm{N}^{\circ}$ 17, IICA, Coronado, Costa Rica, p67.

Ablan, E. 2000. Políticas de Calidad en el Sistema Agroalimentario Español. Agroalimentaria 16(10):63-72.
Aggelogiannopoulos, D., Drosinos, E. y Athanasopoulos, P. 2007. Implementation of a quality management system (QMS) according to the ISO 9000 family in a Greek small-sized winery: A case study. Food Control 18:1077-1085.

Almirall, P., Escobedo, A. y Cimerman, S. 2008. Cyclospora cayetanensis un protozoo intestinal emergente. Revista Panam Infectol 10(1):24-29.

Arenas, A. 2000. El aseguramiento de la calidad e inocuidad de los alimentos. Editorial Retina, Bogotá, p. 273.

Báez, W. 2006. El principio de sistemas aplicado a la cuenca hidrográfica. Memorias II Curso internacional de agroecología. Universidad Pedagógica y Tecnológica de Colombia. Tunja, Agosto de 2006, p356.

Belibagli, K., Vardin, H. y Dalgic, A. 2009. Application of food safetyand quality management systems to bulgur processing. Ital. J. Food Sci. 4(21):499-515.

Bertalanfly, L. 1976. Teoría General de Sistemas. Petrópolis, Vozes, p284.

Borrego, D. 2010. Herramientas para la Mejora Continua, Ciclo Deming. http://www.blogcatalog.com/search. frame=ciclo+deming [4 Marzo 2012].

Cáceres, N. 2004. Nuevos Sistemas Agrarios y su posicionamiento en la cadena agroalimentaria. Informe de la Agricultura Familiar en España, p321. 
CAPLAM. Comisión Latinoamericana de Productividad y Medio Ambiente. 2006. Metodología para la Certificación ISO 9001:2000 de Empresas. http:// www.clapam.com/paginas/4asesoria_ certificacion.htm [26 Junio 2012].

Capra. 1998. La trama de la vida: una nueva perspectiva de los seres vivos. Anagrama. Barcelona. Caps I-III, p286.

Castellanos, O. 2004. Gestión siglo XXI. Nuevas tendencias en la gestión organizacional. www.virtual.unal.edu. co/cursos/económicas/2008551/index. html [16 Julio 2012].

CCI, 2004. Guía de Buenas Prácticas Agrícolas para la producción de hortalizas limpias en la sabana de Bogotá. Servicio Nacional De Aprendizaje, Sena, Bogotá, p59.

\section{Cleves, J., Fonseca, J. y Muñoz, N. 2011. El} sistema de gestión de calidad: elemento para la competitividad y la sostenibilidad de la producción agropecuaria en Colombia. Revista de investigación agraria y ambiental 2(1):9-21.

Codex Alimentarius. 1993. Directrices para la aplicación del Sistema de Análisis de Riesgos y Puntos Críticos (ARCPC). ALINORM 93/13A Apéndice II. NACMCF: National Advisory Committee on Microbiological Criteria for Food. Hazard analysis and critical point system. Revista Internacional de Microbiología de Alimentos 16:1-23.
Codex Alimentarius Commission. 2003. Hazard Analysis and Critical Control Point (HACC P) system and guidelines for its application. En: "Food Hygiene Basic Texts" Food and Agriculture Organization of the United Nations, World Health Organization, Rome, p369.

Codex Alimentarius Commission, Organización Mundial de la Salud y Organización de las Naciones Unidas para la Agricultura y la Alimentación. 2006. Tercera edición. Roma. http:// ftp.fao.org/codex/Publications/ understanding/Understanding_ES.pdf. Consulted [15 Noviembre de 2009].

Conpes, 2001. Atributos de confianza, normas y certificación: comparación de estándares para hortalizas. http://www. infoagro.com [Julio de 2010].

Contreras, A. y Puentes, D. 2009. Diseño del sistema de certificación de fincas ovinas en la provincia norte del departamento de Boyacá. Tesis Administrador de empresas agropecuarias. Universidad Pedagógica y Tecnológica de Colombia. Duitama, p189.

Contreras, U. 2008. El sistema de gestión de la calidad en la empresa agropecuaria. Editorial ECOPANGE Ltda., Bogotá, p73.

Contreras, U. y Restrepo, S. 2007a. Guía de la auditoria en el sistema de control interno para empresas agropecuarias. Editorial ECOPANGEA Ltda., Bogotá, p65. 
Contreras, U.y Restrepo,S. 2007b. Manualde implementación del sistema de control interno como herramienta indispensable para el sector agropecuario. Editorial ECOPANGEA Ltda., Bogotá, p87.

Corpoica, 2010. IV Seminario Nacional sobre avances en Buenas Prácticas Agropecuarias BPA en Colombia y I Encuentro de productores certificados en BPA. Corpoica, Mosquera, septiembre de 2010, p123.

Correa, C. 2009. Normatividad en la Producción Agropecuaria en Colombia: aspectos generales. Universidad Nacional de Colombia. http://www. agro.unalmed.edu.co/departamentos/ panimal/docs/Normatividad.pdf Septiembre 2012].

\section{Deming, E.,Medina, N.y Gozalbes, M. 1989.} Calidad, Productividad y Competitividad: La Salida de la Crisis. Primera edición, Ediciones Díaz de Santos, Madrid, p412.

Espejo, C. 2009. Sistema de Explotación Ganadera. Notas en torno a su concepto. Lurralde. Investigación y Espacio 1(19):89-104.

Fedecacao. 2010. Alternativas de certificación para el cultivo de cacao. Federación Nacional de Cacaoteros, XXVIII Congreso nacional cacaotero. Septiembre 15 -18 2010. Bogotá, p73.

Fernández, L. 2003. "Las certificaciones de calidad. Análisis de riesgos y control de puntos críticos", en Álvarez, J. y
Camacho, F. (coord.) Innovaciones en el Sector Hortofrutícola Español. Secretaría General Técnica del MAPA, Madrid. ISBN84-491-0577-3, p95-113.

FLO, 2002. Overview of Fairtrade certified associations and plantations per country. Información proporcionada por Fairtrade Labelling Organizations International, p49.

Fonseca, J. y Cleves, A. 2010. Manual técnico del cultivo de cebolla puerro (Allium porrum) bajo enfoque de buenas prácticas agrícolas en el distrito de riego del alto chicamocha. Orión editores, Bogotá, p156.

Fonseca, J. y Cleves A. 2009. Los sistemas de gestión de la calidad en la producción de ajo y cebolla en Colombia. Memorias Simposio internacional de cebolla y ajo en el trópico. Paipa (Boyacá), septiembre de 2009, p174.

Fonseca, J. 2007. Contribución al análisis del estado de desarrollo de microempresas agroindustriales del sector lácteo en el corredor central de Boyacá. Tesis Maestría en Desarrollo Empresarial Agropecuario, Universidad Nacional de Colombia, Bogotá.

Gallardo, F. 2000. Pasos para implementar un Sistema de Gestión de la Calidad basado en la norma internacional ISO 9001:2000. http://www.degerencia. com/articulo/pasos_para_implementar_ un_sistema_de_gestion_de_calidad_ basado_en_la_norma_internacional_ ISO 9001 [15 Octubre 2012]. 
GLOBALGAP. 2007. Reglamento General Aseguramiento Integrado De Fincas: Certificación de grupo Cod Ref: IFA 3.01 GR III Versión: V3.0-1July 2007, p3.

Hernández, L. 2009. Certificación Agropecuaria. http://www.ciat.cgiar. org/agroempresas/pdf/certificación [20 Mayo 2010].

IFAT. 2002. Estándares para las organizaciones de Comercio Justo. Versión revisada tras reunión del Comité Ejecutivo de IFAT del 16.02.02. Grupo de trabajo de los estándares, p10.

ISO. 2000a. International Standard ISO 9000:2000. Quality management systems Fundamentals and vocabulary. Geneva: ISO.

ISO. 2000b. International Standard ISO 9001:2000. Quality management systems Requirements. Geneva: ISO.

Manis, M. 1995. The HACCP system and how it fits into FSIS programs. En "HACCP in Meat,Poultry and Fish Processing" (A. M. Pearson and T. R. Dutson, eds.), Blackie Academic and Professional, Londres, p935.

Mateos, M. 2002.SeguridadeHigieneenel sector de frutas y hortalizas. Temas de Actualidad No. 3. AINIA, Instituto Tecnológico Agroalimentario, España, p135.

MinisteriodeAgriculturay DesarrolloRural., SENA. y SAC. 2005. Buenas Prácticas Agrícolas. Produmedios, Bogotá, p70.
Ministerio de Agricultura y desarrollo rural y Corporación Colombiana de investigación agropecuaria. 2007. Guías para la implementación de buenas prácticas ganaderas. Bogotá.

Montañez, S. 2009. Diseño del sistema de certificación de conformidad de producto para BCS Oko garantie. Tesis Administrador de empresas agropecuarias, Universidad Pedagógica y Tecnológica de Colombia, Duitama.

Murcia, H. 2007. Creatividad empresarial para la educación agropecuaria. Segunda edición. Universidad de la Salle, p311.

Linares, N.ySalamanca,Y.2003.Determinación e la capacidad de interacción de las empresas en un ambiente competitivo caso: empresas biotecnológicas. Tesis administradora de empresa. Universidad Nacional de Colombia. Facultad de ciencias económicas y administrativas, Bogotá.

Oyarzún,M.yTartanac,F.2002.Estudiosobre los principales tipos de sellos de calidad en alimentos a nivel mundial. Estado actual y perspectivas de los sellos de calidad en productos alimenticios de la agroindustria rural en América Latina. FAO, Santiago, Chile, p97.

Peinado, J. 2006. El proceso de certificación en la agricultura orgánica y los mercados verdes. Primer diplomado Internacional Agroecología y agricultura orgánica en el trópico, Tunja, p77. 
Pérez, L. 2008. Actualización NOP. http:// www.ocia.org/ResourceCenter/Training/ NOP_SP/7_NOP_Actualizacion_Final. pdf [3 Marzo 2010].

Pop, C. y Pop, I. 2006. Calitate, siguranta si competitivitate prin integrarea sistemelor moderne de management. Lucr. st., seria Zootehni. Editura „Ion Ionescu de la Brad" Iaşip 49:847- 853.

Pop, C., Costin, P., Stef, D. y Mircean, I. 2011. The evolution of non-animal food production sector in lasi county under the european norms on food quality and safety. Lucrări ştiinţifice 54(1):261-266.

Portilla,V.2007.Entornodelacadenaproductiva de las plantas aromáticas, medicinales y condimentarías en Colombia. En: Perspectivas del agronegocio de hierbas aromáticas, culinarias y medicinales. Proyecto Hierbas Aromáticas. Facultad de Agronomía, Universidad Nacional de Colombia, Bogotá p86.

Rooney, J. y Kilkelly, J. 2002. On Today s Menu: Quality Case study shows how HACCP helps ensure farm to tablefood safety. Quality Progress 35(2):25-32.

Ropkins, K. y Beck, A. 2000. Evaluation of worldwide approaches to the use of HACCP to control food safety. Trends in Food Science \& Technology 11:10-21.
Sandrou, D. y Arvanitoyannis, S. 2000. Application of hazard analysis critical control point (HACCP) system to the cheese-making Industry: a review. Food Rev. Int. 16(3):327-368.

Spiegel, M., Luning, P.A., Ziggers, G.W. y Jongen, W. M. F. 2003. Towards a conceptual model to measure efectiveness of food quality systems. Trends in Food Science and Technology 14(10):424-432.

Torrado, A. 2005. Buenas prácticas agrícolas. Sistema de aseguramiento de la inocuidad de los alimentos. Instituto Colombiano Agropecuario. Boletín Técnico. Bogotá. p16.

UTZ Certified good Inside. 2013. Código de conducta para productor. https://www. utzcertified.org/es/sobreutzcertified [19 Marzo 2013].

Venegas, R. 2010. Buenas Prácticas Pecuarias. http://www.faenacar.cl/UserFiles/File/ presentaciones/Rio\%20Verde\% 20 2006/4RaulVenegasProducciónLimpia. pdf [Julio de 2012]. 